\title{
The Urban Heat Island (UHI) is a major source of uncertainty in projections of population-weighted surface temperature under climate change.
}

\author{
Peter Sherman 1 , and Alexander T. Archibald ${ }^{2,3}$ \\ ${ }^{1}$ Department of Earth and Planetary Sciences, Harvard University, Cambridge, \\ Massachusetts 02138, United States. \\ ${ }^{2}$ Department of Chemistry, University of Cambridge, Lensfield Road, Cambridge, UK. \\ ${ }^{3}$ National Centre for Atmospheric Science, UK
}

Corresponding author: Alexander Archibald (ata27@cam.ac.uk)

\begin{abstract}
Rising global mean temperatures and an increase in the fraction of the population living in urban areas is leading to a number of grand challenges. Contrasting areaweighted and population-weighted trends in an ensemble of CMIP5 climate model simulations under a range of climate and population change scenarios (RCPs and SSPs) shows that population-weighted changes in future surface temperature are almost double the area-weighted changes. Furthermore, we show that the Urban Heat Island (UHI) effect can have a large bearing on these calculations. By incorporating and comparing two parsimonious parameterizations of the $\mathrm{UHI}$ we calculate it contributes up-to $56 \%$ of the total uncertainty in population-weighted temperature exposure by the year 2050. However, in the long-term uncertainty in populationweighted temperature is dominated by model and scenario uncertainties rather than the UHI. We suggest that more efforts are required to develop improved parameterizations of the UHI for similar studies in CMIP6.
\end{abstract}

\section{Introduction}

Understanding the risks and impacts of anthropogenic climate change remains one of the most societally important and pressing challenges (National Academies, 2018). Whilst heat-waves (periods of prolonged high temperatures) and heat stress have played a role on population dynamics for centuries (Carleton et al., 2017), several recent studies have shown that climate change will further expose an increasing 
number of people to extreme heat (Hajat et al., 2014; Patz et al., 2005; Meehl and Tebaldi, 2004). A recent study by Mora et al. (2017) found that $74 \%$ of the world's population could be exposed to deadly climate conditions by 2100 . Coffel et al., (2017) have calculated that there will be 150-750 million person-days of exposure to wet bulb temperatures (the temperature of a fully saturated parcel that has been evaporatively cooled) above those seen in today's most severe heat waves by 2070-2080. As large as these numbers may seem, they may be underestimates; Mora et al. (2017) and Coffel et al. (2017) excluded the urban heat island (UHI) effect in their calculations. The UHI is the surface temperature difference between urban areas and the surrounding rural areas. The UHI was first observed as early as the 1880 s when data from London and surrounding areas were analyzed and revealed temperature biases in the city relative to the surrounding countryside (Wilby et al., 2011). Whilst a simple concept, the causes are due to several complex factors including changing land surfaces and waste heat from anthropogenic activity, building geometries and materials and many more (e.g. building asymmetry, Wang et al., 2017). Regardless of the complexity in causing the $\mathrm{UHI}$, it is important now and is likely to become increasingly important as it can have a significant impact on local temperatures and human exposure to extreme heat (Heaviside et al., 2017). Jones et al. (2008) calculate that the UHI effect can be on the order of $4-5{ }^{\circ} \mathrm{C}$ for daily mean temperatures. Continued urbanization has seen a rise in the number of people exposed to the UHI. Since 2007, more than $50 \%$ of the global population live in urban areas (World Bank, 2017). Recent projections of population change (Jones \& O'Neill, 2016) indicate that this fraction will increase throughout the coming decades meaning that more and more people are likely to be affected by the impacts of the UHI (Heaviside et al., 2017). It is therefore imperative to compute the impacts of accounting for the $\mathrm{UHI}$ on population wide exposure to temperature for future climate and population change scenarios to see if the UHI has an important as yet overlooked effect.

In this study we aim to quantify the impacts of the UHI on projections of surface temperature change. Our aim is not to quantify the exposure of future populations to heat stress or extreme temperature events, but rather to quantify the extent the UHI has on projections of temperature change which are population-weighted. Here we focus on the broader impacts of the UHI and population change in assessments of temperature change. We make use of the concept of population-weighting 
temperature change as a fairly novel metric which we show has bearings in how we communicate temperature change with climate models. There is a large literature on the topics and metrics of heat stress and exposure (e.g. Buzan et al., 2015) and we refer the reader to this for more detailed discussion.

\section{Materials and Methods}

To investigate the impacts of the UHI on simulations of future population-weighted temperature changes, we combined monthly-mean surface temperature data from models participating in the CMIP5 project (Taylor et al., 2012), which contributed to the IPCC AR5 (IPCC, 2014), and considered climate and population projections dating from 2010 to 2100. Future climate projections in CMIP5 were modelled with Representative Concentration Pathways (RCPs) for greenhouse gases and future population distributions follows the Shared Socioeconomic Pathways (SSPs) (Jones \& O’Neill 2016).

The RCPs represent different climate scenarios determined by future greenhouse gas emissions and their impacts on radiative forcing. Four RCP scenarios were investigated in this study, defined by $2.6,4.5,6.0$ and $8.5^{\circ} \mathrm{C} \mathrm{Wm}^{-2}$ of climate forcing by the end of the century. Simulations of these RCP scenarios were taken from six models: MIROC-ESM (Watanabe et al., 2011), MIROC-ESM-CHEM (Watanabe et al., 2011) (MIROC-ESM's chemically-coupled counterpart), MRI-CGCM3 (Yukimoto et al., 2012), IPSL-CM5 (Dufresne et al., 2013), NorESM (Bentsen et al., 2013) and GISS (Nazarenko et al., 2015), a subset of the models which ran the RCP scenarios in aid of the CMIP5 intercomparison. These models were chosen as a subset of the CMIP5 ensemble. The monthly mean surface temperature data from the four RCPs in each model were used to calculate decadal mean values from 2010 to 2100 to combine with SSP population data. To understand how future changes will be affected by the UHI we chose 2010 as a starting point as it is a point where data are readily available for.

The SSPs represent five potential future population pathways that vary due to different narratives of development. SSP1 is defined as a "sustainability" scenario, SSP2 is "middle of the road", SSP3 is "regional rivalry", SSP4 is "inequality" and SSP5 is "fossilfuel development" (Jones and O'Neill, 2016). Each of these scenarios is assigned different numerical values to several key factors including population growth and 
urbanization level. These scenarios output spatial projections of urban and rural populations every 7.5 arcminutes at a temporal resolution of a decade.

To assess the impacts of changes in surface temperature on population we re-gridded both these population and climate model data onto a uniform grid at a 7.5 arcminute resolution. The land surface schemes in climate models (which run at resolutions of several $100 \mathrm{~km}^{2}$ ) generally try to account for urbanization by including urban tiles (e.g. Best et al., 2011). Such schemes describe the detailed thermodynamical processes associated with the urban land-form and are capable of simulating the UHI (Oleson 2012). Very recently, work with the Community Land Model (CLM) (Oleson et al., 2015) has enabled quantification of exposure to heat extremes accounting for the UHI in simulations using the CESM model (Jones et al., 2018). But in general, these schemes are more common place in the current generation of climate models than those used in CMIP5 and future work looking at the representation of the UHI in climate models that participate in CMIP6 is warranted. At the low resolution they are used in climate models, these schemes still suffer from limitations in simulating the UHI sufficiently well to capture the observed enhancements in surface temperature that the UHI causes (e.g. Jones et al., 2008). To rectify this issue, we implemented three different UHI measures as bias correction factors to the CMIP5 climate model data to enable us to determine the impact of the $\mathrm{UHI}$ on future population-weighted temperature exposure. Since more than $51 \%$ of the world's population live in urban areas, we would expect the $\mathrm{UHI}$ to be a significant factor in population-weighted temperature under climate change.

Three sets of UHI data were employed in this study. The first was taken as a reference dataset; the Global Urban Heat Island (UHI) Data Set v1 2013, a Columbia University product derived from the Aqua Level-3 Moderate Resolution Imaging Spectroradiometer (MODIS) (CIESIN, 2016). This dataset represents a 30 arcsecond gridded index of $2013 \mathrm{UHI}$ values, which were calculated from the measured difference in temperature between urban areas (as defined by SEDACs Global RuralUrban Mapping Project) and the surrounding $10 \mathrm{~km}$. We refer to this as the UHISAT product. It should be noted that the Global Rural-Urban Mapping Project (GRUMP) used in the generation of this UHI dataset is known to overestimate the areal extent of 
urban land (Zhou et al., 2015), potentially leading to a low bias in the UHI product we are using.

The other two data sets were used in our analysis of the time evolution of the impacts of the UHI and derived from an observed power-law relationship between UHI index and population (Karl et al., 1988). The UHI is calculated as a function of time $t$ for a given cell (lat, lon), based on the size of the urban population in the cell $P_{U R B}(t$, lat,lon):

$$
U H I(t, \text { lat,lon })=a \times P_{U R B}(t, \text { lat }, \text { lon })^{b},
$$

where $a$ is $2.07 \times 10^{-3}$ for populations less than $10,000,1.51 \times 10^{-3}$ for populations between 10,000 and 100,000 and $1.74 \times 10^{-3}$ for populations greater than 100,000 and $b$ is 0.45 , as derived from an analysis of $\mathrm{UHI}$ over cities of varying population in the USA (Karl et al., 1988). For a more detailed explanation of the methodology, we refer the reader to Karl et al. (1988). These equations were used to form our second $\mathrm{UHI}$ index at a 7.5 arcminute resolution, referred to as the UHIPOP product. The final metric was formed by performing a regression of the UHISAT product to an urban population dataset for 2010 (Jones and O'Neill, 2016) to derive best-fit coefficients of $a$ and $b$ and produced a merged product, UHISP. The parameters $a$ and $b$ were found not to vary much with respect to population size, so only one regression was performed, which found values of $4.99 \pm 0.76 \times 10^{-1}$ for $a$ and $8.94 \pm 1.71 \times 10^{-2}$ for $b$. UHIPOP and UHISP follow the different SSPS and vary with time as the SSP urban populations were time-dependent. The resolutions of all temperature and UHI data were (re)gridded to 7.5 arcminutes to compare with the population data. These data were then used to calculate the population-weighted temperature, $T_{P W} . T_{P W}$ is defined as

$$
T_{P W}(t, \text { lat }, \text { lon })=\frac{(T(t, \text { lat }, \text { lon })+U H I(\text { lat }, \text { lon })) P_{U R B}(t, \text { lat }, \text { lon })+T(t, \text { lat }, \text { lon }) P_{R U R}(t, \text { lat }, \text { lon })}{P_{U R B}+P_{R U R}},
$$

where $T$ (t,lat,lon) is the model cell's (RCP dependent) temperature at a given time, $U H I$ (lat,lon) is the cell's UHI index and $P_{U R B}\left(t\right.$, lat,lon) and $P_{R U R}(t$,lat,lon) are the cell's urban and rural population, respectively, and change in space and time according to the SSPs (Jones and O'Neill, 2016). TPW was then calculated with and without UHIPOP and $\mathrm{UHI}_{\mathrm{SP}}$ for each RCP and SSP, yielding a total of 240 population-temperature scenarios projected out to the year 2100. Comparisons of the UHISAT to UHIPOP and UHISAT to UHISP are shown in Figure 1. 



Figure 1. Comparison of the satellite-based UHI against (a) the population-based UHI and (b) UHIsP for the year 2013. The color bar denotes the density of points with yellow colored hexagons representing a greater density of points than blue hexagons. It should be noted that a subset of the UHIPOP and UHISP datasets are used here since several cells with a population datapoint did not also have a satellitebased UHI datapoint. For simplicity, negative-valued satellite-based UHI data were excluded. A 1-to-1 line is included in red for reference.

The UHIPOP and UHIsP scenarios can be compared with the no UHI scenarios to assess the relative importance of including the $\mathrm{UHI}$ and, following Hawkins and Sutton (2009), to quantify the uncertainty that arises from adding the UHI compared to the inherent model and scenario uncertainties that affect population-weighted temperature. The model uncertainty is defined as the response of different models to the same radiative forcing, which can be expressed as $\frac{1}{N_{R C P}} \sum_{R C P} \operatorname{var}\left(x_{R C P, S S P}\right)$, where $N_{R C P}$ is the number of RCPs and $x_{R C P, S S P}$ is the data from a specific RCP and SSP. The scenario uncertainty represents other factors that could influence the populationweighted temperature, and can be expressed as $\operatorname{var}\left(\frac{1}{N_{R C P}} \sum_{R C P}\left(x_{R C P, S S P}\right)\right)$.

\section{Results}

In order to first determine the fidelity of UHIPOP and UHISP we compare the grid cells from these two datasets for the year 2010 (all SSPs are very similar for this decade) against the same grid cells in UHISAT for 2013. Scatter plots were made to assess the 
fit of UHIPOP and UHISP to UHISAT (Figure 1, panels (a) and (b), respectively). There is a relatively low but positive correlation between $U H I_{P O P}(R=0.22)$ and $U H_{S P}(R=$ 0.24 ) with UHISAT. This can be seen geographically in these data (not shown): most of the largest UHISAT values are located on the east coasts of the United States and Asia, while they can primarily be seen in India and north Africa for both UHIPOP and UHISP. In terms of numerical discrepancies, the $5^{\text {th }} / 95^{\text {th }}$ percentile range of UHISAT is 0.09 to $2.53{ }^{\circ} \mathrm{C}$ whilst the same range for the UHIPOP is 0.02 to $0.35{ }^{\circ} \mathrm{C}$ and 0.82 to $1.43{ }^{\circ} \mathrm{C}$ for UHISP. In general, UHIPOP underestimates the satellite-based data, with a bias of 0.93 ${ }^{\circ} \mathrm{C}$. While UHIPOP does not adequately parameterize the observed UHI, it can be treated as a lower bound for the $\mathrm{UHI}$ because of this underestimation. $\mathrm{UHI}_{\mathrm{SP}}$ underestimates minimally with a mean bias of $5.49 \times 10^{-4}{ }^{\circ} \mathrm{C}$. From Figure 1, panel (b), we can however see that most of the data is overestimated by UHISP as it lies below the 1-to-1 line. The conclusions drawn further about the inclusion of UHIPOP and UHISP on the potentially large impact of including the UHI should be treated as likely lower and upper bounds on the effects.
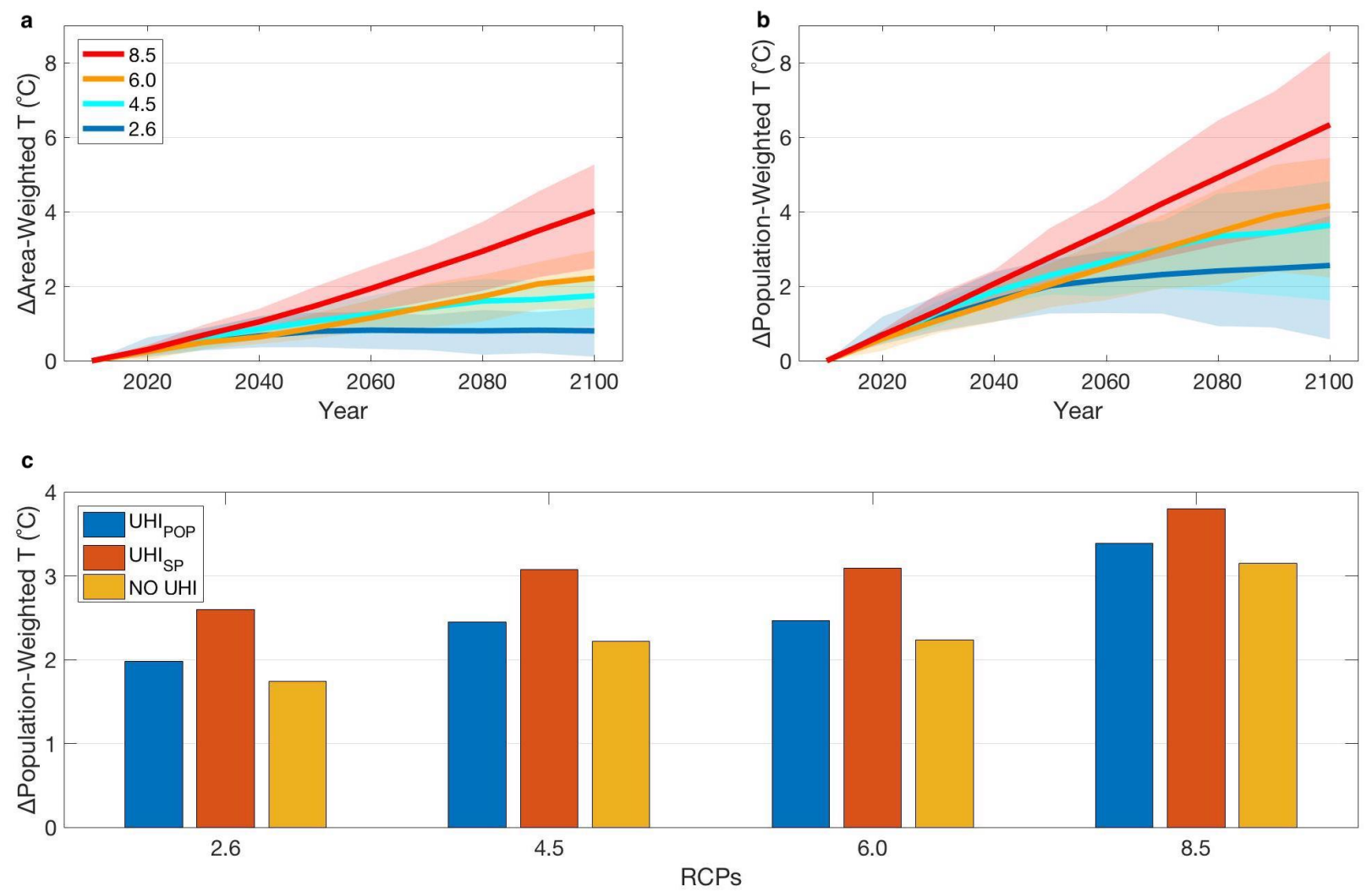

Figure 2. Time series of the change in (a) area-weighted temperature and (b) population-weighted temperature (in ${ }^{\circ} \mathrm{C}$ ) for the range of different models, RCP and SSP scenarios relative to 2010 values. The solid lines represent the mean of each RCP and the transparent fills are the upper and lower 
bounds. The changes in population-weighted temperature for each RCP are shown for the populationbased UHI, UHIsp and no UHI scenarios in panel (c).

Figure 2 contrasts the changes we calculate with the CMIP5 models in area and population-weighted decadal mean temperatures over the period 2010-2100, using 2010 values as a baseline. Analysis of Figure 2 panel (a) shows that the limited ensemble mean we have assessed captures the trends in area-weighted changes in surface temperature in good agreement with the full CMIP5 ensemble means (IPCC, 2014). Figure 2 panel (a) follows very similarly Figure 1 from Knutti and Sledacek (2012), albeit with changes in temperature relative to a different base year. This provides confidence that our subset of the CMIP5 models reproduce the key features amongst the different RCPs. However, a key difference can be seen in Figure 2 between the area- and population-weighted temperature values we calculate, with the population-weighted values changing much more dramatically with time. Accounting for population-weighting leads to surface increases in temperature which are more than double those if the area-weighting is only considered. At a very basic level, comparing Figure 2 panel (a) and (b) shows that when it comes to climate change, people should expect much larger change at the personal scale than the global surface average indicates, perhaps unsurprising but something we have not found quantification of in the literature before. Indeed, even under the most optimistic climate scenario (RCP2.6) population-weighted temperature change at the end of the $21^{\text {st }}$ century could reach up to $4^{\circ} \mathrm{C}$ compared with at the start of the century.

Figure 2 panel (c) shows, as we hypothesized, that a discrepancy is seen between the different scenarios (with and without UHI); the mean difference between UHIPOP and no UHI temperature scenarios over the period 2010 to 2100 is $0.24{ }^{\circ} \mathrm{C}$ and similarly $0.80{ }^{\circ} \mathrm{C}$ between $\mathrm{UHISP}$ and no $\mathrm{UHI}$ scenarios. These figures and results indicate that the population-based UHI may have a significant effect on global population-weighted surface temperature. 

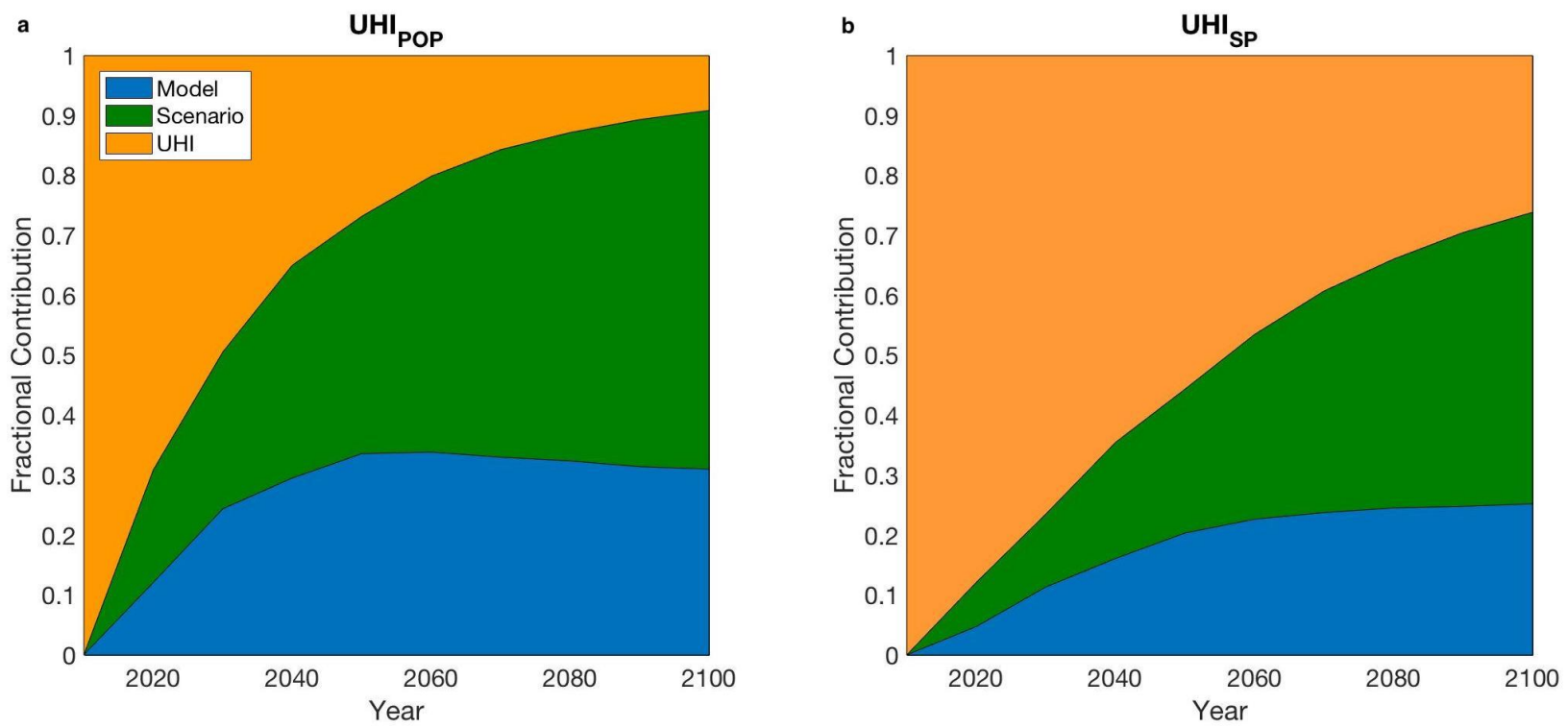

Figure 3. Time series of the fractional contributions from different sources of uncertainty for the population-weighted temperature for (a) the population-based UHI and (b) UHIsP.

To further assess this conclusion, we compared the uncertainty from UHIPOP and UHIsp to the other uncertainties that were inherent to the models themselves (Figure 3). In the short-term, both UHIPOP and UHIsP are relatively important, and actually dominate the other uncertainties until 2030 for UHIpop and 2050 for UHIsp. After that time, however, the population-weighted temperature exposure is dominated by model and scenario uncertainties (both SSPS and RCPs). It is interesting to note that the uncertainty in population-weighted temperature exposure from including the UHIPOP or UHIsp remains relatively flat throughout the century, around $0.24{ }^{\circ} \mathrm{C}$ or $0.80{ }^{\circ} \mathrm{C}$, respectively, while the model and scenario uncertainties grow strongly with time. This is consistent with similar calculations on surface mean temperature, although this is the first study to include the UHI (Lehner and Stocker, 2015). This result is further indication that the $\mathrm{UHI}$ has a potentially noteworthy impact on the exposure of the population to temperature.

\section{Conclusions}

The main purposes of this study were to quantify the effects of looking at global climate change at the human scale, by weighting changes in surface temperature proportional to population, and to evaluate and assess the impacts of including two representations of the $\mathrm{UHI}$ for projections of population-weighted temperature based on data of future 
projections of temperature change from global climate models and population change from SSP scenarios. We found that population-weighted temperature exposure follows the surface mean temperature exposure, increasing with time and radiative forcing (Lehner and Stocker, 2015), but is much stronger - up to a factor of two more larger in magnitude. We further found that a population-weighted UHI index is an important factor to consider in the short-term when using results from global climate model projections. In the long term, climate model and scenario uncertainties dominate uncertainty in calculations of population-weighted temperature exposure and so the importance of the UHI drops off as being a principal factor for estimating an upper bound on population-weighted temperature.

It should be noted that the population-based UHI contains more data-points because the population dataset (SSP scenarios) classifies a larger region as "urban" than the satellite-based data, particularly over central Africa. What qualifies as an urban area can have a significant effect on the population-weighted temperature change attributed to the UHI, and this discrepancy between the two datasets therefore illustrates a potential flaw in our study. Urban definitions essentially fall between those being based on population density and those based on land-form. Our UHI dataset (CIESIN, 2016) being satellite and so land-form derived, typically will cover an area smaller than one based on population density (i.e. the definition used in Jones and O'Neil, 2016). This has the implication that our results may underestimate the true impact of the UHI. To guide future work, a clearer definition of an urban population should be made in order to more accurately model impacts over these regions. However, we note this topic remains one of great debate in the demographics community.

A major limitation remains in the development and application of robust parameterizations of the UHI that can be used with future population change datasets. As can be seen in Figure 1, the UHls included in this study (as defined by Karl et al. (1988)) do not capture satellite-based estimates of the UHI for the near present day. Can a parsimonious expression do this? We think that new data-based approaches, such as Machine/Deep Learning could provide breakthroughs in this area. However, we believe that this does not have significant bearing on our main conclusions; while these UHIs may not give an excellent fit to the satellite based UHI, we have seen that 
these data may actually provide upper and lower bounds on the intensity of the UHI. This would confirm our conclusion that global and local climate model simulations of surface temperature can be affected by the UHI. To further verify this, alternative parametrization should be investigated. This alternative parametrization should include construction materials, human behavior, air pollutants and other factors as identified in Wang et al. (2017). These variables could vary drastically under different SSPs. In the sustainable scenario, SSP1, for example, we might expect a reduced UHI due to improved building standards. Such additional factors are nonetheless beyond the scope of this paper, the purpose of which was to demonstrate the necessity of including the effects of the UHI in climate models to assess the implications for surface temperature under future climate change.

\section{Acknowledgments, Samples, and Data}

We thank two anonymous reviewers and Dr Scott Archer-Nicholls for useful feedback which has helped improve this paper. ATA thanks NERC and NCAS for funding through the ACSIS project, which has supported various elements of this work. We acknowledge the WCRP's Working Group on Coupled Modelling, which is responsible for CMIP, and we thank the climate modeling groups (listed in Table S1 of this paper) for producing and making available their model output. For CMIP the U.S. Department of Energy's Program for Climate Model Diagnosis and Intercomparison provided the coordinating support and led development of software infrastructure in partnership with the Global Organization for Earth System Science Portals. CMIP5 data can be accessed at https://esgfnode.Innl.gov/projects/cmip5/. We also acknowledge Center for International Earth Science Information Network - CIESIN - Columbia University. 2016. Global Urban Heat Island (UHI) Data Set, 2013. Palisades, NY: NASA Socioeconomic Data and Applications Center (SEDAC) http://dx.doi.org/10.7927/H4H70CRF. Accessed December 2016. 


\section{References}

Bentsen, M., Bethke, I., Debernard, J. B. et al. (2013). The Norwegian Earth System Model, NorESM1-M - Part 1: Description and basic evaluation of the physical climate, Geosci. Model Dev., 6, pp.687-720.

Best, M. J., Pryor, M., Clark, D.B et al. (2011). The Joint UK Land Environment Simulator (JULES), model description-Part 1: energy and water fluxes. Geoscientific Model Development 4.3 pp.677-699.

Buzan, J. R., K. Oleson, and M. Huber (2015), Implementation and comparison of a suite of heat stress metrics within the Community Land Model version 4.5, Geosci. Model Dev., 8(2), 151-170, doi:10.5194/gmd-8-151-2015.

Carleton, W.C., Campbell, D. \& Collard, M. (2017). Increasing temperature exacerbated Classic Maya conflict over the long term. Quaternary Science Reviews, 163, pp.209-218.

Center for International Earth Science Information Network - CIESIN - Columbia University. 2016. Global Urban Heat Island (UHI) Data Set, 2013. Palisades, NY: NASA Socioeconomic Data and Applications Center (SEDAC). [Accessed 26 July 2017].

Coffel, E.D., Horton, R. \& de Sherbinin, A. (2017). Temperature and humidity based projections of a rapid rise in global heat stress exposure during the $21 \mathrm{st}$ century. Environ. Res. Lett., 13, 1, 014001.

Dufresne, J.-L., Foujols, M.-A., Denvil, S., et al. (2013). Climate change projections using the IPSL-CM5 earth system model-From CMIP3 to CMIP5. Climate Dynamics, 40, 9, pp. 2123-2165.

Hajat, S., Vardoulakis, S., Heaviside, C. et al. (2014). Climate change effects on human health: projections of temperature-related mortality for the UK during the 2020s, 2050s and 2080s. J Epidemiol Community Health.

Hawkins, E. \& Sutton, R. (2009). The potential to narrow uncertainty in regional climate predictions. Bull. Am. Meteorol. Soc., 90(8), pp.1095-1107.

Heaviside, C., Macintyre, H., \& Vardoulakis, S. (2017). The Urban Heat Island: Implications for Health in a Changing Environment. Current Environmental Health Reports 1-10.

IPCC, 2014: Climate Change 2014: Synthesis Report. Contribution of Working Groups I, II and III to the Fifth Assessment Report of the Intergovernmental Panel on 
Climate Change [Core Writing Team, R.K. Pachauri and L.A. Meyer (eds.)]. IPCC, Geneva, Switzerland, pp.151.

Jones, B. \& O'Neill, B.C. (2016). Spatially explicit global population scenarios consistent with the Shared Socioeconomic Pathways. Environmental Research Letters 11, 84003.

Jones, B., Tebaldi, C., O’Neill, B. C., Oleson, K., \& Gao, J. (2018). Avoiding population exposure to heat-related extremes: demographic change vs climate change. Climatic Change, 146(3-4), 423-437.

Jones, P.D., Lister, D.H. \& Li, Q. (2008). Urbanization effects in large-scale temperature records, with an emphasis on China J. Geophys. Res. Atmos. 113 D16122.

Karl, T., Diaz, H. \& Kukla, G. (1988). Urbanization: Its Detection and Effect in the United States Climate Record. Journal of Climate, 1(11), pp.1099-1123.

Knutti, R., and J. Sedláček, 2012: Robustness and uncertainties in the new CMIP5 climate model projections. Nat. Climate Change, 3, 369-373.

Lehner, F. \& Stocker, T.F. (2015). From local perception to global perspective. Nature Climate Change, 5, pp.731-734.

Meehl, G. A. \& Tebaldi, C. (2004). More intense, more frequent, and longer lasting heat waves in the 21st century. Science 305, 994-997.

Mora, C., Dousset, B., Caldwell, I., et al. (2017). Global risk of deadly heat. Nature Climate Change 7(7), pp.501-506.

National Academies of Sciences, Engineering, and Medicine. (2018). Thriving on Our Changing Planet: A Decadal Strategy for Earth Observation from Space. Washington, DC: The National Academies Press.

Nazarenko, L., Schmidt, G.A., Miller, R.L. et al. (2015). Future climate change under


pp.244-267.

Oleson, K. (2012), Contrasts between Urban and Rural Climate in CCSM4 CMIP5 Climate Change Scenarios, J. Climate, 25(5), 1390-1412, doi:10.1175/JCLI-D11-00098.1.

Oleson K.W., Anderson G.B., Jones B. et al (2015) Avoided climate impacts of urban and rural heat and cold waves over the U.S. using large climate model ensembles for RCP8.5 and RCP4.5. Clim Chang. https://doi.org/10.1007/s10584-015-1504-1 
Patz, J. A., Campbell-Lendrum, D., Holloway, T. et al. (2005). Impact of regional climate change on human health. Nature 438, 310-317.

Taylor, K.E., Stouffer, R.J., \& Meehl, G.A. (2012). An overview of CMIP5 and the experiment design. Bulletin of the American Meteorological Society 93.4 pp.485-498.

Wang, K., Li, Y., Wang, Y. \& Yang, X. (2017). On the asymmetry of the urban daily air temperature cycle. Journal of Geophysical Research: Atmospheres.

Watanabe, S., Hajima, T., Sudo, K. et al. (2011). MIROC-ESM: model description and basic results of CMIP5-20c3m experiments. Geoscientific Model Development Discussions, 4(2), pp.1063-1128.

Wilby, R.L., Jones, P.D., Lister D.H. (2011). Decadal variations in the nocturnal heat island of London. Weather 66.

The World Bank. (2017). Urban population (\% of total) | Data. [online] Available at: https://data.worldbank.org/indicator/SP.URB.TOTL.IN.ZS?page=4. [Accessed 21 Aug. 2017].

Yukimoto, S., Adachi, Y., Hosaka, M. et al. (2012). A New Global Climate Model of the Meteorological Research Institute: MRI-CGCM3 Description and Basic Performance. Journal of the Meteorological Society of Japan, 90A(0), pp.2364.

Zhou Y., et al. (2015). A global map of urban extent from nightlights. Environmental Research Letters 10.5 (2015): 054011. 